\title{
LA ECONOMÍA SOCIAL ANTE LA ACTUAL CRISIS ECONÓMICA EN LA COMUNIDAD AUTÓNOMA DEL PAÍS $\operatorname{VASCO} \mathrm{O}^{1}$
}

\author{
POR \\ Ignacio BRETOS FERNÁNDEZ ${ }^{2} \mathrm{y}$ \\ Jon MORANDEIRA ARCA ${ }^{3}$
}

\section{RESUMEN}

El presente artículo analiza la evolución socio-económica del conjunto de entidades que configuran el sector de la economía social en el País Vasco durante el periodo de crisis comprendido entre 2008 y 2014. Los datos empleados se han obtenido en un marco de colaboración entre universidad, administración pública y sector privado de la economía social. Los resultados sugieren que este tipo de organizaciones han evolucionado mejor, tanto a nivel del número de empresas como de empleo, que la economía general del País Vasco, a pesar de que el contexto de las políticas públicas dirigidas a la economía social se ha visto reducido durante estos años. Sin embargo, en términos económicos (medido a través del Valor Añadido Bruto generado), no han sido capaces de hacer frente a la crisis en iguales condiciones que el conjunto de la economía general. La principal contribución de la investigación presentada reside en que, a diferencia de estudios similares, se analiza la evolución de todas las entidades enmarcadas en la economía social tomando como referencia un amplio periodo temporal de la crisis actual.

\footnotetext{
${ }^{1}$ Estudio realizado en el marco del Proyecto de Investigación "Observatorio Vasco de Economía Social Gizarte Ekonomiako Euskal Behatokia (OVES/GEEB)" del Instituto GEZKI de Derecho Cooperativo y Economía Social de la Universidad del País Vasco/Euskal Herriko Unibertsitatea, financiado por la Dirección de Economía Social del Departamento de Empleo y Políticas Sociales del Gobierno Vasco.

${ }_{2}^{2}$ Departamento de Economía Aplicada I. Instituto GEZKI de Derecho Cooperativo y Economía Social Universidad del País Vasco/Euskal Herriko Unibertsitatea. Dirección de correo electrónico (disponible para correspondencia): ignaciobretos@gmail.com.

${ }^{3}$ Departamento de Economía Financiera II. Instituto GEZKI de Derecho Cooperativo y Economía SocialUniversidad del País Vasco/Euskal Herriko Unibertsitatea. Dirección de correo electrónico: jon.morandeira@ehu.eus.
}

REVESCO No 122 - Tercer Cuatrimestre 2016 - ISSN: 1885-8031 - www.ucm.es/info/revesco

http://dx.doi.org/10.5209/rev_REVE.2016.v122.52020

Fecha de recepción: 06/01/2016

Fecha de aceptación: 15/02/2016 
Palabras clave: Economía social, Crisis Económica, Comunidad Autónoma del País Vasco, Densidad Empresarial, Empleo, Valor Añadido Bruto.

Claves ECONLIT: C810, D220, E320, J210.

\title{
SOCIAL ECONOMY AND ECONOMIC CRISIS IN THE BASQUE COUNTRY
}

\begin{abstract}
This article examines the socio-economic evolution of the social economy sector in the Basque Country during the 2008-2014 period of economic crisis. Data have been obtained within a framework of collaboration between university, Basque Government and private sector of the social economy. The results suggest that such entities have evolved better, both in terms of number of enterprises and employment, than the general economy of the Basque Country, while the context of public policies aimed at social economy has worsened over the years. However, in economic terms (measured through the Gross Value Added generated), they have not been able to cope with the crisis in equal conditions to the general economy. The main contribution of this research lies in that, unlike similar studies, it discusses the evolution of the whole sector of the social economy, taking as reference a broad period of the current economic crisis.
\end{abstract}

Keywords: Social Economy, Economic Crisis, Basque Country, Number of Enterprises, Employment, Gross Value Added.

\section{INTRODUCCIÓN}

Las cooperativas y otras entidades que forman parte de la economía social han sido ampliamente consideradas, tanto desde un punto de vista académico como institucional, como elementos estratégicos para fomentar un crecimiento inteligente, sostenible e integrador en el entorno actual (Monzón, 2013) y fortalecer el desarrollo local de los territorios (Williamson et al., 2003). Especialmente en coyunturas de recesión económica, este tipo de organizaciones responden en mejores condiciones que las empresas convencionales (Birchall y Ketilson, 2009), al generar empleo estable y fortalecer la cohesión económica y social (Chaves y Monzón, 2012; Castro et al, 2013), mostrando así un comportamiento contrario al ciclo económico en periodos de recesión (Grávalos y Pomares, 1991). 
En este sentido, un amplio número de estudios, tanto a nivel internacional como a nivel de España y de algunas comunidades autónomas, han analizado el comportamiento de la economía social durante distintas fases contractivas de la economía. Sin embargo, es habitual encontrar en estos trabajos generalizaciones para el conjunto de la economía social aun cuando se analizan exclusivamente alguna de sus fórmulas, como cooperativas o sociedades laborales. Asimismo, muchos estudios miden la respuesta de estas entidades a la crisis económica únicamente en términos de número de empresas y/o de empleo, sin considerar su evolución económica y contribución a la economía.

Tomando en consideración estos elementos, el presente artículo realiza un análisis transversal de la evolución socio-económica (número de empresas, empleo y contribución económica) durante el periodo de crisis 2008-2014 del conjunto de entidades que forman el sector de la economía social vasca, y se compara en términos análogos con la evolución experimentada por la economía general de la Comunidad Autónoma del País Vasco (CAPV en adelante). El análisis de este territorio cuenta con fuertes potencialidades para obtener unas conclusiones sólidas sobre el comportamiento de la economía social en la actual recesión económica, ya que es una región con una presencia significativa de las distintas experiencias que se encuadran en este sector.

Los resultados obtenidos demuestran que el sector de la economía social ha respondido mejor a la crisis que el conjunto de la economía general en la CAPV, especialmente en términos de empleo, a pesar de que el contexto de políticas públicas dirigidas al sector ha empeorado a lo largo de la crisis. Asimismo, ponen en relieve dos cuestiones fundamentales. Por un lado, la importancia de contar con una conceptualización sólida acerca de la economía social que abarque de manera integradora el conjunto de entidades que conforman su sector, ya que se ha observado que su evolución ha sido más positiva que si tomamos como referencia exclusivamente a cooperativas y/o sociedades laborales. Por otro lado, la necesidad de disponer de estadísticas homogéneas, completas y continuadas sobre la economía social ya que, presumiblemente, se habrían obtenido unos resultados incluso más contundentes que los que aquí se presentan.

El artículo se estructura de la siguiente manera. Tras este apartado introductorio, se discute la conceptualización y delimitación de la economía social y su relevancia en el contexto de crisis actual. En el tercer apartado se realiza una breve revisión de la literatura principal sobre la respuesta de la economía social ante coyunturas de recesión económica. En 
el cuarto apartado se presenta el estudio empírico sobre el comportamiento de la economía social vasca durante la actual crisis, explicando detalladamente la metodología y datos empleados. El quinto apartado destaca las principales conclusiones del artículo recogiendo las limitaciones y contribuciones de la investigación, remarca algunas necesidades en materia de política pública y plantea líneas de investigación futura.

\section{DELIMITACIÓN CIENTÍFICA DE LA ECONOMÍA SOCIAL Y SU RELEVANCIA EN EL CONTEXTO DE CRISIS}

\subsection{La importancia de la Economía Social en contextos de crisis}

La economía social ha recibido en los últimos años una notable atención institucional, no sólo como sector estratégico para promover una salida justa y social de la crisis económica, sino como un actor clave en la consecución de los objetivos de la estrategia Europa 2020 (Monzón, 2013; Monzón y Chaves, 2012: 90-107). En este sentido, mientras el sector público y el sector privado capitalista están experimentando serias dificultades en muchos países, las cooperativas y otras entidades de la economía social están mostrando una mayor estabilidad y capacidad de respuesta en estos años de crisis (Stiglitz, 2009; Birchall, 2013), demostrando en definitiva un marcado carácter anti-cíclico (Grávalos y Pomares, 1991; Bartlett y Pridham, 1991; Westerdahl y Westlund, 1998).

Ello se debe, entre otros aspectos, a la menor dependencia de estas empresas en los mercados financieros y a la naturaleza socializada de su capital, lo que les ha permitido sufrir una menor presión en la crisis financiera (Birchall y Ketilson, 2009). Asimismo, su orientación social y su gobernanza inclusiva tienen un impacto positivo en el fortalecimiento de la cohesión social y en la consecución de un crecimiento económico sostenible (Stiglitz, 2009; Birchall, 2010; Castro et al., 2013; Chaves y Monzón, 2012) basado en el desarrollo local de los territorios y el bienestar de las comunidades (Williamson et al., 2003; Buendía y García, 2003). Elementos clave como la capacidad para crear y mantener empleo estable y de calidad (Perotin, 2013; Burdin y Dean, 2009), para hacer frente a la desigualdad en los ingresos (Ben-ner et al., 2011), o para proveer bienes y servicios públicos (Erdal, 2011, 2012; Thomas, 2004), juegan igualmente un papel fundamental en los efectos positivos que generan estas organizaciones a nivel social.

Debido a estas cuestiones, las instituciones públicas están reconociendo e integrando el papel de la economía social en la consecución de sus objetivos. Entre las iniciativas más 
recientes destacan, por ejemplo, la Resolución del Parlamento Europeo, de 2 de julio de 2013, sobre la contribución de las cooperativas a la salida de la crisis, en la que se destaca el papel esencial de las cooperativas y otras empresas de la economía social en la economía europea, especialmente en tiempos de crisis (Parlamento Europeo, 2013). Por otra parte, el nuevo programa marco de la UE para el empleo y la política social (EaSi), desarrollado por la Comisión Europea, pone en relieve las potencialidades de la economía social y las empresas sociales para contribuir a la consecución de las iniciativas emblemáticas y objetivos de Europa 2020 (Comisión Europea, 2013).

La Resolución del Parlamento Europeo, de 10 de septiembre de 2015, sobre emprendimiento social e innovación social en la lucha contra el desempleo, sitúa a la economía social y solidaria como motor para el empleo y la inclusión social en Europa frente a la crisis (Parlamento Europeo, 2015). También es de destacar la reciente resolución del Consejo Europeo, de 7 de diciembre de 2015, en la que se reconoce que la economía social es un sector que ha capeado la crisis económica mucho mejor que otros sectores, contribuyendo sustancialmente al desarrollo económico, social y humano de Europa y a alcanzar los objetivos clave de la UE: crecimiento inteligente, sostenible e integrador, empleo de alta calidad, cohesión social, innovación social, desarrollo local y regional, y protección del medio ambiente (Consejo Europeo, 2015).

\subsection{La delimitación científica de la Economía Social: una visión desde País Vasco}

En el ámbito de la delimitación teórica de la economía social, CIRIEC-Internacional es la referencia utilizando como base los Sistemas de Contabilidad Nacionales en vigor (SCN1993 y SEC-1995). CIRIEC proporciona la definición de la Economía Social más reciente y con mayor aval institucional, al estar basada en un amplio consenso político y científico (Monzón, 2010a, 2010b), y aceptada por el CESE-Comité Económico y Social Europeo. La definición propuesta en dicho informe (Chaves y Monzón, 2008: 20; Monzón y Chaves, 2012: 23) delimita la economía social en sus dos vertientes, dividiéndose así en dos subsectores: de mercado, donde operan las entidades más tradicionales, y de no mercado, donde operan organizaciones sin ánimo de lucro (Pérez de Mendiguren et al., 2009; Etxezarreta y Morandeira, 2012).

El subsector de mercado está conformado por productores de mercado, que hace referencia a aquéllos cuyo destino principal es la venta en el mercado a precios 
económicamente significativos ${ }^{4}$ (Chaves y Monzón, 2008; Monzón y Chaves, 2012). Existe un principio que se establece como distintivo y discriminante más allá de la forma jurídica de cada organización: la gestión democrática tanto en los órganos de decisión ("una persona, un voto"), como en el reparto de beneficios en su caso (Pérez de Mendiguren et al., 2009). El subsector de no mercado está conformado por otros productores no de mercado, que hace referencia a aquéllos que suministran su producción a otras unidades de forma gratuita o a precios económicamente no significativos ${ }^{5}$. Se incluyen todas las entidades del "Tercer Sector de acción social" que, aún no teniendo una estructura democrática, suministran bienes preferenciales o de interés general (Chaves y Monzón, 2008; Monzón, 2010a, 2010b).

En España, Monzón (2010a, 2010b) realiza una minuciosa delimitación conceptual de las entidades que forman parte de la economía social en España. Se adopta la definición planteada en el informe, subrayando que el criterio democrático es imprescindible para los productores de mercado, ya que la utilidad social de los mismos no se apoya habitualmente en la actividad económica desarrollada sino que proviene de su propia finalidad y de sus valores democráticos y participativos. En cuanto a las instituciones sin fines de lucro, Monzón (2010a: 51, 2010b: 55) afirma que "aunque la gran mayoría de ellas tienen un funcionamiento democrático, desarrollan actividad económica y reúnen las características propias de la Economía Social, la oportunidad de su inclusión en el ámbito de la misma está sometida a debate”. Por ello, Monzón (2010c) distingue entre entidades de acción social y entidades cuya finalidad es la de encuentro y ocio, influencia o representación que, aunque tienen una indudable utilidad social, su inclusión está sometida a debate.

Por tanto, tal y como se aprecia, CIRIEC apuesta por una definición que "debe ignorar los criterios jurídicos y administrativos y centrarse en analizar el comportamiento de los actores de la ES y detectar las similitudes y las diferencias entre ellos y entre estos y otros agentes económicos" (Monzón y Chaves, 2012: 23). Esta postura tiene su raíz en la irrupción de nuevas realidades y conceptos afines, tales como las empresas sociales y la economía solidaria (Pérez de Mendiguren et al., 2009; Etxezarreta y Morandeira, 2012), y que incluso se ha visto reflejada en el ámbito institucional/legal con la formulación de la Ley 5/2011 (Etxezarreta y Morandeira, 2012).

\footnotetext{
${ }^{4}$ Cuando las ventas de la producción suponen un mínimo del 50\% de los costes de producción.

${ }^{5}$ Aquéllos en los que las ventas suponen menos del 50\% del coste de producción.
} 
En el País Vasco, el Observatorio Vasco de Economía Social (OVES/GEEB), nacido en el seno del Instituto de Derecho Cooperativo y Economía Social (GEZKI) de la Universidad del País Vasco (UPV/EHU) - miembro a su vez de CIRIEC-España y CIRIECInternacional - asume la definición realizada por la misma adecuándola a las características propias de la economía social en el País Vasco. Así, determina la economía social vasca tal y como se muestra en la figura 1, la cual será objeto de estudio en la presente investigación.

Figura 1. Mapa de las entidades de la Economía Social vasca.

\begin{tabular}{|c|c|c|}
\hline Subsector de la Economía Social & Sector Institucional & Organización microeconómica \\
\hline \multirow[t]{2}{*}{ Productores de mercado } & Sociedades no financieras & $\begin{array}{l}\text { - } \text { Cooperativas } \\
\text { - } \text { Sociedades Laborales } \\
\text { - Empresas de Inserción } \\
\text { - } \text { Centros Especiales de Empleo } \\
\text { - } \text { Sociedades Agrarias de Transformación } \\
\text { - } \text { Cofradías de Pescadores } \\
\text { - Otros productores de mercado privados } \\
\text { (asociaciones, fundaciones y sociedades } \\
\text { mercantiles) }\end{array}$ \\
\hline & Sociedades financieras & $\begin{array}{l}\text { - Cooperativas de Crédito } \\
\text { - } \quad \text { Entidades de Previsión Social } \\
\text { Voluntaria } \\
\text { - Sociedades de Garantía Recíproca de } \\
\text { entidades de Economía Social }\end{array}$ \\
\hline Productores de no mercado & $\begin{array}{l}\text { Instituciones sin fines de } \\
\text { lucro al servicio de los } \\
\text { hogares (ISFLSH) }\end{array}$ & $\begin{array}{l}\text { - } \quad \text { Asociaciones de acción social } \\
\text { - } \quad \text { Fundaciones de acción social } \\
\text { servicio de los hogares }\end{array}$ \\
\hline
\end{tabular}

Fuente: Observatorio Vasco de Economía Social (OVES-GEEB).

\section{REVISIÓN DE LA LITERATURA: EL COMPORTAMIENTO DE LA ECONOMÍA SOCIAL EN PERIODOS DE CRISIS ECONÓMICA}

Un amplio número de estudios se han centrado en estudiar empíricamente el comportamiento de algunas tipologías de entidades de la Economía Social (especialmente cooperativas y/o sociedades laborales) en periodos de crisis económica, fundamentalmente en términos del número de empresas y del empleo que aglutinan. Por un lado, diversos estudios sugieren que en coyunturas económicas negativas las cooperativas son capaces de mantener e incluso aumentar el número de sus empresas (Ben-ner, 1988; Conte y Jones, 1991), debido, entre otros aspectos, a que se erigen como respuestas de auto-organización para las personas en paro (Spear, 1996) y como vehículos para la recuperación de empresas en quiebra por parte de los trabajadores (Bhowmik y Sarker, 2000; Vieta, 2013). Por otro lado, la literatura se ha centrado en la capacidad de estas organizaciones para mantener y generar empleo en 
situaciones de crisis económica (Roelants et al., 2014; Estrin, 1985) con condiciones sociolaborales, además, superiores a los de otras empresas convencionales del entorno (Smith y Rothbaum, 2013; Perotin, 2013), así como en su capacidad para lograr la inclusión sociolaboral de colectivos en riesgo de exclusión (Melián y Campos, 2010; Marcuello, 2008; Barea y Monzón, 2008).

De esta manera, a nivel internacional, Perotin (2006) compara los patrones de entrada y salida de un amplio conjunto de empresas convencionales y de cooperativas de trabajo asociado francesas entre 1971 y 2002, demostrando que se crean más cooperativas cuando las tasas de desempleo aumentan y el crecimiento económico es más lento, al igual que cuando las tasas de interés y las expectativas de beneficios son más bajas. Fakhfakh et al. (2012) comparan para el periodo 1987-2004 el comportamiento de una gran muestra de empresas convencionales y sociedades cooperativas en Francia. Entre otras conclusiones, destacan que las cooperativas francesas son tan eficientes como las empresas convencionales, y además el empleo en las cooperativas muestra un comportamiento más estable en los ciclos de mayor inestabilidad económica.

Carini y Carpita (2014) analizan la respuesta del sector cooperativo industrial italiano en un momento de fuerte crisis económica como es el periodo 2008-2010, tomando como referencia una muestra de 25.000 empresas industriales (300 cooperativas). La conclusión más relevante que obtienen es que, a pesar de que el número de cooperativas se reduce durante el periodo, el nivel de empleo se mantiene más o menos estable, mientras que en el resto del sector industrial se continúa destruyendo puestos de trabajo.

Rondinelli y Sarateanu (2012) estudian la evolución de las diversas tipologías de cooperativas en diferentes países europeos. Por ejemplo, en el caso de Reino Unido, se muestra una tasa de crecimiento del sector cooperativo del 15,1\% durante el periodo 20082010, registrándose aumentos en todas las tipologías de cooperativas y mostrando así un mejor comportamiento que la economía convencional. Asimismo, las cooperativas británicas han presentado incrementos continuados durante el periodo de crisis 2009-2013 en las cifras globales de facturación, empleo y número de socios (Co-operatives UK, 2014), mientras que en el conjunto de la economía se ha producido una destrucción de empleo durante dicho periodo. 
Zevi et al. (2011) analizan el comportamiento durante la crisis actual de cooperativas francesas, italianas y españolas de diversos sectores y concluyen que, en términos generales, estas entidades han logrado hacer frente a las consecuencias de la crisis de manera más eficiente que las empresas tradicionales privadas, destacando especialmente que han sido capaces de mantener los niveles de empleo.

En el caso concreto de España, Román (2014) analiza si los ciclos de creación de las cooperativas guardan relación con los periodos de expansión o contracción de la economía española. Tomando como referencia diversas tipologías de cooperativas y un amplio periodo temporal, comprendido entre los años 40 y el inicio del siglo XXI, concluye que, si bien estas organizaciones respondían positivamente a los estímulos de los mercados, fue en las etapas de escaso crecimiento del PIB cuando las cooperativas en su conjunto alcanzaron un mayor dinamismo, demostrando así un perfil anticíclico.

En el reciente estudio de Sala et al. (2014) sobre el comportamiento cíclico de las cooperativas y sociedades laborales españolas durante el periodo 1995-2013, se constata que ambas entidades, aun con ciertas diferencias, resisten mejor el impacto de las fases recesivas en términos de mantenimiento y creación de empresas. Sin embargo, como señalan, esta capacidad de supervivencia no se traslada en la misma medida al empleo, detectándose un comportamiento pro-cíclico en el caso de las sociedades laborales españolas durante la actual recesión económica y un cierto acoplamiento al ciclo económico en el caso de las cooperativas. Díaz y Marcuello (2010), si bien no demuestran un efecto refugio del sector cooperativo español entre 1994 y 2008, sí encuentran una menor dependencia del empleo cooperativo ante las variaciones del Producto Interior Bruto. Así, confirman que en fases recesivas del PIB la destrucción de empleo en las cooperativas es menor que en el conjunto de la economía.

En términos más amplios, Calderón y Calderón (2012), tomando como referencia el año 2009 en un contexto de fuerte recesión económica, analizan la calidad del empleo en las cooperativas, sociedades laborales y centros especiales de empleo españoles. Concluyen que los efectos de la crisis económica han sido menores sobre este tipo de entidades que sobre las empresas convencionales. Asimismo, señalan que dichas entidades se caracterizan por generar un empleo de calidad gracias a aspectos como la igualdad de género, la estabilidad del empleo, la flexibilidad del empleo, la participación activa de los trabajadores, o la inclusión laboral y no discriminación. 
En definitiva, estos trabajos sugieren que las entidades de la economía social analizadas son capaces de resistir las situaciones de crisis económica en mejores condiciones que otras empresas convencionales de su entorno. Sin embargo, se puede apreciar que habitualmente sólo se toma como referencia la evolución de cooperativas y/o sociedades laborales (y raramente de otros tipos de entidades como los centros especiales de empleo), mientras que se generalizan en muchas ocasiones los resultados hablando en términos generales de economía social, a pesar de que otras tipologías de empresas conforman también este sector. De esta manera, existen pocos trabajos que realmente analicen la respuesta del conjunto de entidades de la economía social ante la crisis actual en términos de número de empresas, empleo y contribución económica. La investigación presentada a continuación trata de contribuir en esta dirección.

\section{ESTUDIO EMPÍRICO: COMPORTAMIENTO DE LA ECONOMÍA SOCIAL VASCA ANTE LA ACTUAL CRISIS ECONÓMICA}

\subsection{Metodología}

En el presente estudio se realiza un análisis comparativo para el periodo 2008-2014 de la evolución de la economía social y de la economía general en la CAPV, empleando tres indicadores: evolución del número de empresas, del empleo y del valor añadido bruto (VAB). La evolución económica se ha medido a través del VAB ya que este indicador permite evaluar el valor económico generado por las unidades productivas que forman parte de la economía social y medir la contribución económica de la economía social en el conjunto de la economía general vasca (Gobierno Vasco, 2012).

Los datos aquí presentados se han obtenido en el marco del proyecto de investigación "Observatorio Vasco de Economía Social” del Instituto GEZKI de la Universidad del País Vasco/ Euskal Herriko Unibertsitatea, dentro de una relación de colaboración entre universidad, administración pública y sector privado de la economía social. En este sentido, se han empleado diversas fuentes de información, las cuales se pueden agrupar en tres tipos: 1) fuentes públicas y oficiales, como es el caso por ejemplo del Ministerio de Empleo y Seguridad Social; 2) fuentes de las asociaciones representativas de entidades de la economía social como, por ejemplo, GIZATEA- Asociación de Empresas de Inserción del País Vasco; y 3) fuentes de las propias entidades de la economía social como es el caso, por ejemplo, de la única sociedad de garantía recíproca incluida en la economía social vasca, Oinarri S.G.R. En 
los cuadros presentados en el presente apartado empírico se especifican las fuentes de información concretas que se han empleado para obtener cada dato.

Sin embargo, es de destacar que existen diversos obstáculos para disponer de datos completos, homogéneos y continuados sobre el conjunto de la economía social en el País Vasco (Bretos, 2015), que no han permitido realizar un análisis completo para el periodo 2008-2014. Por ello, en función de los datos disponibles, se ha optado por dividir el análisis en dos niveles. Un primer nivel toma como referencia lo que hemos denominado Economía Social Principal, que integra a las entidades con mayor peso dentro del sector de la economía social vasca: cooperativas, sociedades laborales, centros especiales de empleo y empresas de inserción (en 2012, el empleo de estas entidades representaba el 81,77\% del total de la economía social vasca) ${ }^{6}$. A nivel de empleo y número de empresas se dispone de datos completos para todos los años del periodo 2008-2014. Sin embargo, a nivel del VAB, sólo se dispone de datos completos para los años 2008, 2010 y 2012. Por otra parte, un segundo nivel toma como referencia lo que hemos denominado el Sector Total de la Economía Social, que integra al conjunto de entidades que conforman la economía social vasca. En este caso, se dispone de datos completos a nivel de empleo, número de empresas y VAB para los años 2010 y 2012, por lo que se analizará la evolución experimentada entre dichos años.

\subsection{Evolución del número de empresas}

Como se observa en la siguiente tabla 1, las cooperativas han mantenido, en general, una evolución estable, e incluso muestran un leve aumento en términos absolutos entre 2008 y 2014. Sólo se presentan descensos en los años 2009 y 2014, mientras que en el resto de anualidades se experimentan variaciones positivas. Las sociedades laborales, en cambio, muestran una evolución negativa durante todo el periodo temporal, aunque en 2013 y especialmente en 2014, se observa una tendencia hacia la menor destrucción de empresas. Respecto a los centros especiales de empleo, a pesar de que su número de empresas es reducido, muestran una evolución positiva durante el periodo, llegando a doblar el número de empresas entre 2008 y 2014. Por su parte, las empresas de inserción muestran un ligero aumento en términos absolutos entre 2008 y 2014, a pesar de que en 2009 y 2014 se producen leves descensos en el número de empresas.

\footnotetext{
${ }^{6}$ También es significativo que las federaciones representativas de estas cuatro familias (Konfekoop, ASLE, EHLABE y GIZATEA) han creado la Red Vasca de Economía Social (El País, 2015).
} 
Tabla 1. Evolución del número de empresas en la Economía Social y en la Economía General de la CAPV. Periodo anual $2008-2014$.

\begin{tabular}{|c|c|c|c|c|c|c|c|}
\hline & 2008 & 2009 & 2010 & 2011 & 2012 & 2013 & 2014 \\
\hline Cooperativas (1) & 1.511 & 1.478 & 1.498 & 1.522 & 1.533 & 1.536 & 1.532 \\
\hline Sociedades Laborales (1) & 931 & 864 & 815 & 762 & 709 & 674 & 659 \\
\hline Centros Especiales de Empleo (2) & 6 & 6 & 6 & 8 & 9 & 11 & 12 \\
\hline Economía Social Principal CAPV & 2.490 & 2.389 & 2.360 & 2.336 & 2.296 & 2.267 & 2.248 \\
\hline Variación anual & - & $-4,06 \%$ & $-1,21 \%$ & $-1,02 \%$ & $-1,71 \%$ & $-1,26 \%$ & $-0,84 \%$ \\
\hline Variación 2010-2012 & & & & $-2,71 \%$ & & & \\
\hline Sociedades de Garantía Recíproca (4) & 1 & 1 & 1 & 1 & 1 & 1 & 1 \\
\hline Entidades de Previsión Social Voluntaria (5) & n.d. & n.d. & 175 & n.d. & 191 & n.d. & 179 \\
\hline Sociedades Agrarias Transformación (6) & 111 & 108 & 105 & 102 & 101 & 96 & 95 \\
\hline Cofradías de Pescadores (7) & 16 & 16 & 16 & 16 & 16 & 16 & 16 \\
\hline Asociaciones de Utilidad Pública (8) & n.d. & n.d. & 185 & n.d. & 189 & n.d. & n.d. \\
\hline Fundaciones (8) & n.d. & n.d. & 585 & n.d. & 615 & n.d. & n.d. \\
\hline $\begin{array}{l}\text { Sector Total de la Economía Social } \\
\text { Variación 2010-2012 }\end{array}$ & - & - & 3.427 & $\begin{array}{c}- \\
-0,53 \%\end{array}$ & 3.409 & - & - \\
\hline Representación & - & - & $2,05 \%$ & - & $2,11 \%$ & - & - \\
\hline Economía general CAPV (9) & 179.953 & 178.062 & 167.270 & 161.658 & 161.626 & 157.405 & 155.306 \\
\hline Variación anual & - & $-1,05 \%$ & $-6,06 \%$ & $-3,36 \%$ & $-0,02 \%$ & $-2,61 \%$ & $-1,33 \%$ \\
\hline Variación 2010-2012 & & & & $-3,37 \%$ & & & \\
\hline
\end{tabular}

(1) Fuente: Ministerio de Empleo y Seguridad Social, Gobierno de España.

(2) Fuente: EHLABE - Asociación de Entidades de Trabajo Protegido del País Vasco.

(3) Fuente: GIZATEA- Asociación de Empresas de Inserción del País Vasco.

(4) Fuente: Oinarri, S.G.R.

(5) Fuente: Dirección de Política Financiera y Recursos Institucionales, Departamento de Hacienda y Finanzas, Gobierno Vasco.

(6) Fuente: Dirección de Agricultura y Ganadería, Departamento de Desarrollo Económico y Competitividad, Gobierno Vasco.

(7) Fuente: Federación de Cofradías de Pescadores de Guipúzcoa y Federación de Cofradías de Pescadores de Vizcaya.

(8) Fuente: Dirección de Economía Social, Departamento de Empleo y Políticas Sociales, Gobierno Vasco (2010, 2012).

(9)Economía General CAPV: Número Total de Empresas de la Comunidad Autónoma del País Vasco. Fuente: EUSTAT-Instituto Vasco de Estadística.

REVESCO No 122 - Tercer Cuatrimestre 2016 - ISSN: 1885-8031 - www.ucm.es/info/revesco 
Comparando la evolución de la Economía Social Principal y de la economía general de la CAPV (gráfico 1), se observa en primer lugar que ambas evoluciones están marcadas por tasas negativas que se traducen en la destrucción de empresas. Sin embargo, excepto en 2009 y 2012, la Economía Social Principal ha respondido mejor que el conjunto de la economía general de la CAPV, presentando variaciones negativas significativamente menores que las experimentadas por la Economía General.

Gráfico 1. Comparativa de las variaciones porcentuales en el número de empresas de la Economía Social Principal y de la Economía General de la CAPV (2008-2014).

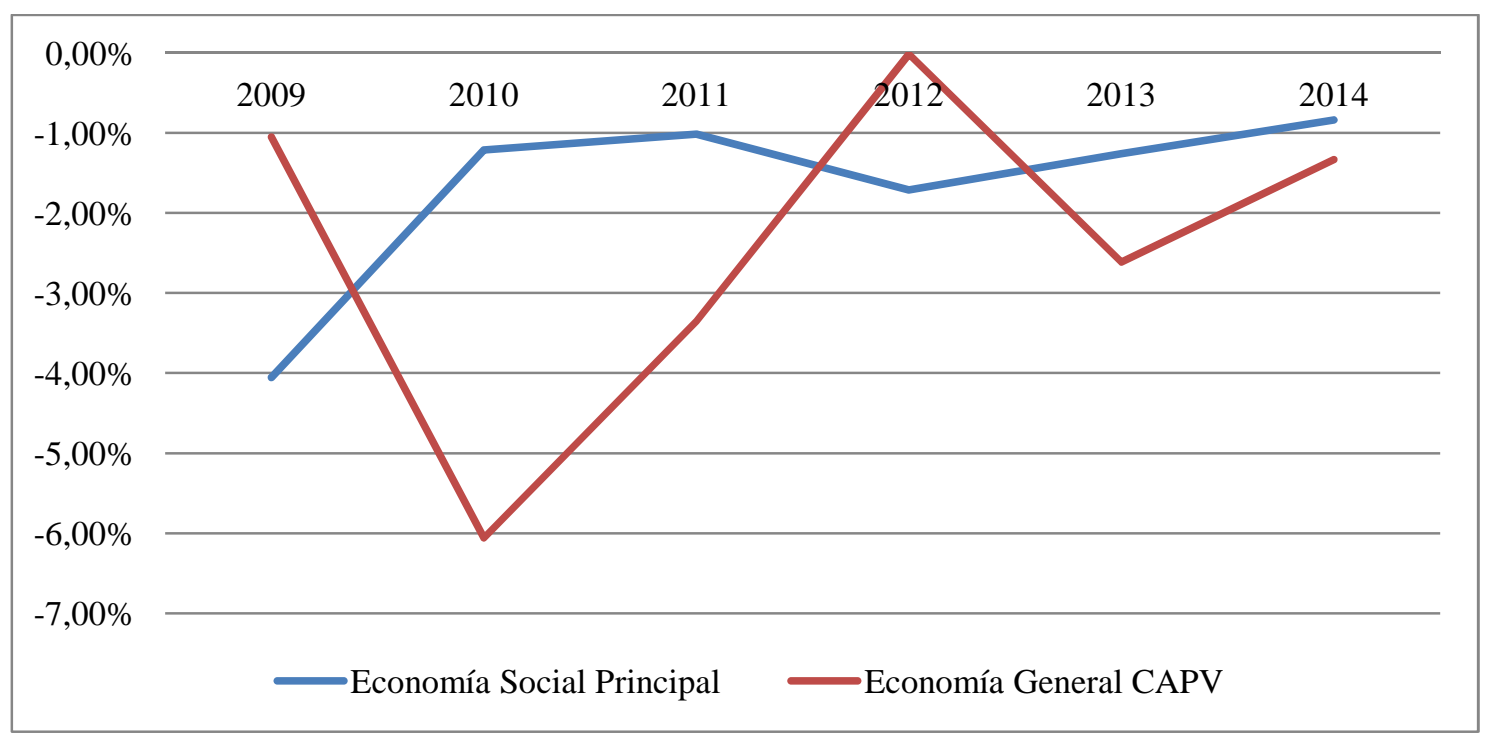

Fuente: elaboración propia

Tomando como referencia la evolución del Sector Total de la Economía Social vasca, se puede observar que entre 2010 y 2012, años en los que se dispone de datos completos y homogéneos para el conjunto de entidades de la economía social, se ha producido un descenso del $0,53 \%$ en el número de empresas de economía social, dato claramente influenciado por la mala evolución de las sociedades laborales. Asimismo, destaca que este descenso del $0,53 \%$ es significativamente menor que el descenso del 2,71\% experimentado por la Economía Social Principal entre 2010 y 2012.

Nuevamente, comparando con la evolución de la economía general vasca, se observa que el Sector Total de la Economía Social ha sido capaz de mantener mejor el número de empresas ante la crisis entre 2010 y 2012 con un descenso del 0,53\% frente al 3,37\%. Ello se traduce en que la representación del número de empresas de economía 
social dentro del conjunto de la economía general de la CAPV ha aumentado ligeramente del $2,05 \%$ al $2,11 \%$.

\subsection{Evolución del empleo}

A raíz de los datos presentados en la siguiente tabla 2, destaca que las cooperativas no han sido capaces de mantener el nivel de empleo entre 2008 y 2014, sufriendo tasas de destrucción de empleo en todos los años excepto en 2010 y 2011. Las sociedades laborales muestran variaciones porcentuales negativas entre 2008 y 2013, mientras que en el año 2014 se produce un cambio de tendencia con un leve repunte del 2,45\%. En cualquier caso, entre 2008 y 2014, las sociedades laborales han perdido casi la mitad de empleos. Los centros especiales de empleo han experimentado descensos en el empleo en 2009 y 2012. Sin embargo, en términos generales, han mostrado una capacidad extraordinaria para generar empleo durante el periodo de crisis. Por su parte, la evolución del empleo en las empresas de inserción fue muy positiva en los primeros años de crisis 2009 y 2010, pero en años posteriores se ha incurrido en tasas de variación negativas. Aun así, en términos absolutos, han sido capaces de generar empleo ligeramente entre 2008 y 2014.

Si comparamos la evolución de la Economía Social Principal y de la economía general de la CAPV (gráfico 2), se observa que ambas evoluciones se caracterizan generalmente por la destrucción de empleo entre 2008 y 2014. Nuevamente, excepto en 2009 y 2012 la Economía Social Principal ha respondido mejor a la crisis en términos de empleo que el conjunto de la economía general de la CAPV.

REVESCO No 122 - Tercer Cuatrimestre 2016 - ISSN: 1885-8031 - www.ucm.es/info/revesco 
Gráfico 2. Comparativa de las variaciones porcentuales en el empleo de la Economía Social Principal y de la Economía General de la CAPV (2008-2014).

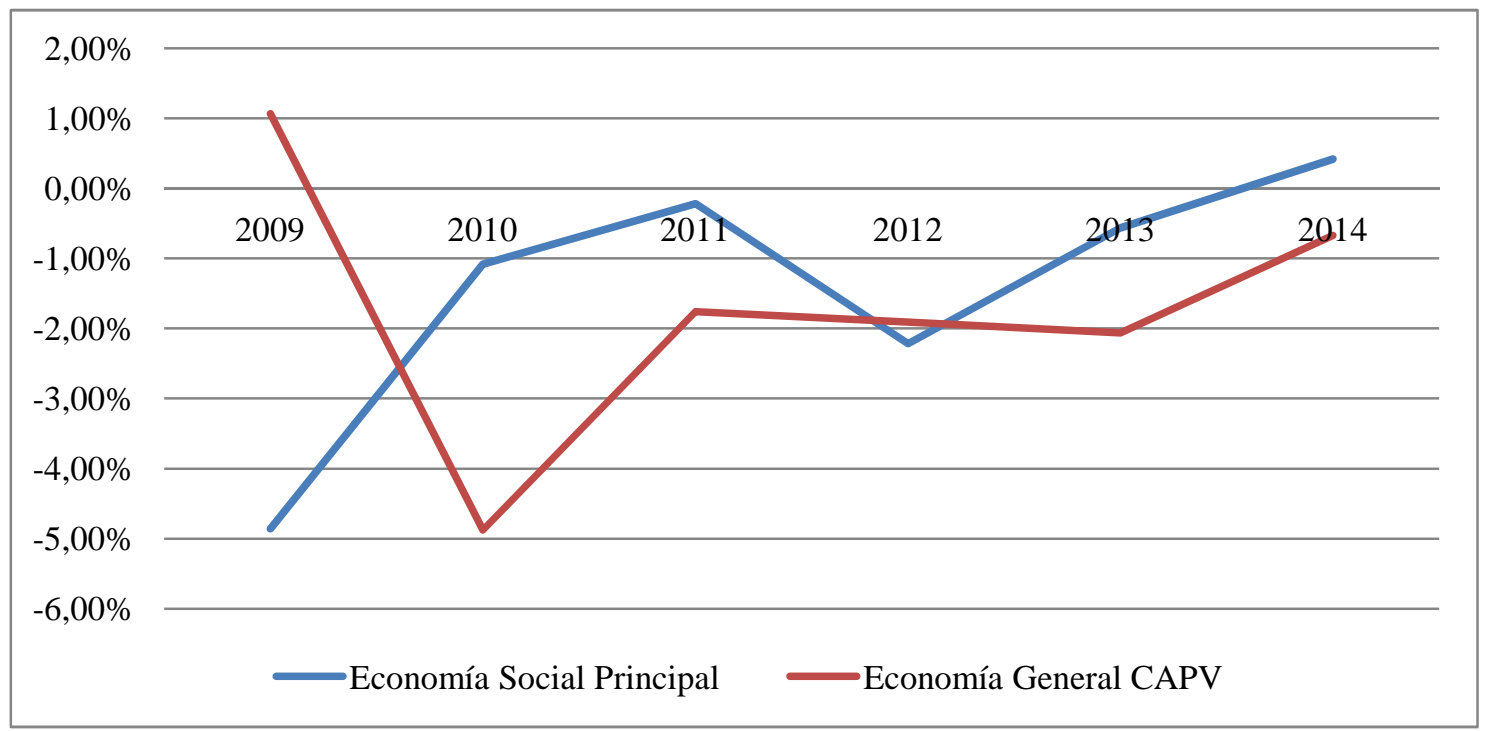

Fuente: elaboración propia 
Tabla 2. Evolución del número de empleos en la Economía Social y en la Economía General de la CAPV. Periodo anual 2008-2014.

\begin{tabular}{|c|c|c|c|c|c|c|c|}
\hline & 2008 & 2009 & 2010 & 2011 & 2012 & 2013 & 2014 \\
\hline Cooperativas (1) & 57.512 & 55.121 & 56.231 & 57.122 & 56.365 & 56.251 & 55.958 \\
\hline Sociedades Laborales (1) & 12.565 & 11.444 & 9.177 & 7.945 & 7.275 & 6.519 & 6.679 \\
\hline Centros Especiales de Empleo (2) & 6.778 & 6.395 & 6.646 & 6.858 & 6.786 & 7.231 & 7.746 \\
\hline Economía Social Principal CAPV & 77.394 & 73.634 & 72.837 & 72.677 & 71.063 & 70.662 & 70.958 \\
\hline Variación anual & - & $-4,86 \%$ & $-1,08 \%$ & $-0,22 \%$ & $-2,22 \%$ & $-0,56 \%$ & $0,42 \%$ \\
\hline Variación 2010-2012 & & & & $-2,44 \%$ & & & \\
\hline Sociedades de Garantía Recíproca (4) & 13 & 19 & 19 & 19 & 20 & 20 & 21 \\
\hline Entidades de Previsión Social Voluntaria (5) & n.d. & n.d. & 93 & n.d. & 99 & n.d. & 95 \\
\hline Sociedades Agrarias Transformación (6) & n.d. & n.d. & 104 & n.d. & 248 & n.d. & n.d. \\
\hline Cofradías de Pescadores (7) & 84 & 82 & 86 & 80 & 75 & 71 & 68 \\
\hline Asociaciones de Utilidad Pública (6) & n.d. & n.d. & 2.235 & n.d. & 3.075 & n.d. & n.d. \\
\hline Fundaciones (6) & n.d. & n.d. & 10.213 & n.d. & 12.315 & n.d. & n.d. \\
\hline $\begin{array}{l}\text { Sector Total de la Economía Social } \\
\text { Variación 2010-2012 }\end{array}$ & - & - & 85.587 & $1,53 \%$ & 86.895 & - & - \\
\hline Economía general CAPV (8) & 942.479 & 952.510 & 906.044 & 890.093 & 873.121 & 855.093 & 849.335 \\
\hline Variación anual & - & $1,06 \%$ & $-4,88 \%$ & $-1,76 \%$ & $-1,91 \%$ & $-2,06 \%$ & $-0,67 \%$ \\
\hline Variación 2010-2012 & & & & $-3,63 \%$ & & & \\
\hline
\end{tabular}

(1) Fuente: Ministerio de Empleo y Seguridad Social, Gobierno de España.

(2) Fuente: EHLABE - Asociación de Entidades de Trabajo Protegido del País Vasco.

(3) Fuente: GIZATEA- Asociación de Empresas de Inserción del País Vasco.

(4) Fuente: Oinarri, S.G.R.

(5) Fuente: Dirección de Política Financiera y Recursos Institucionales, Departamento de Hacienda y Finanzas, Gobierno Vasco.

(6) Fuente: Dirección de Economía Social, Departamento de Empleo y Políticas Sociales, Gobierno Vasco (2010, 2012).

(7) Fuente: Federación de Cofradías de Pescadores de Guipúzcoa y Federación de Cofradías de Pescadores de Vizcaya.

(8) Economía General CAPV: Número Total de Empleos en la Comunidad Autónoma del País Vasco. Fuente: EUSTAT-Instituto Vasco de Estadística.

REVESCO No 122 - Tercer Cuatrimestre 2016 - ISSN: 1885-8031 - www.ucm.es/info/revesco 
Analizando la evolución del Sector Total de la Economía Social vasca entre 2010 y 2012, se observa una evolución positiva del empleo especialmente significativa en las sociedades agrarias de transformación, asociaciones y fundaciones, mientras que los peores datos son nuevamente los presentados por las sociedades laborales. En definitiva, destaca que se ha producido un incremento en el empleo del 1,53\% en el Sector Total de la Economía Social, mientras que entre los mismos años, la Economía Social Principal ha perdido un 2,44\% del empleo.

Asimismo, comparando con la evolución de la economía general vasca, se observa que el empleo en la CAPV ha caído entre 2010 y 2012 un 3,63\%, dato significativamente peor que la evolución experimentada tanto por la Economía Social Principal $(-2,44 \%)$ como por el Sector Total de la Economía Social $(+1,53)$. Este escenario se traduce en que el empleo de la economía social ha pasado de representar el 9,45\% del empleo total de la CAPV en 2010 a casi el 10\% en 2012.

\subsection{Evolución del Valor Añadido Bruto (VAB)}

En relación al valor añadido bruto (VAB) generado por la economía social vasca, debido a los obstáculos que ya señalábamos anteriormente, sólo se dispone de datos completos sobre la Economía Social Principal para los años 2008, 2010 y 2012. Por tanto, a pesar de que se presenten todos los datos que se han podido obtener, se realizará exclusivamente un análisis de la evolución experimentada entre dichos periodos.

Así, tomando en consideración los datos presentados en la siguiente tabla 3, se observa que las cooperativas han sido capaces de aumentar su capacidad de generación de VAB en términos absolutos entre 2008 y 2012, con una variación extraordinaria de algo más del $20 \%$ entre 2008 y 2010, aunque también con un descenso de casi el $6 \%$ entre 2010 y 2012. Por el contrario, en la línea de la evolución presentada en términos del número de empresas y del empleo, las sociedades laborales presentan descensos continuados en el VAB generado en los años 2008, 2010 y 2012. Por otra parte, los centros especiales de empleo y las empresas de inserción muestran una evolución positiva en el VAB generado con incrementos significativos entre 2008 y 2010, así como entre 2010 y 2012. 
Tabla 3. Evolución del Valor Añadido Bruto generado por la Economía Social y por la Economía General de la CAPV. Periodo $2008-2014$.

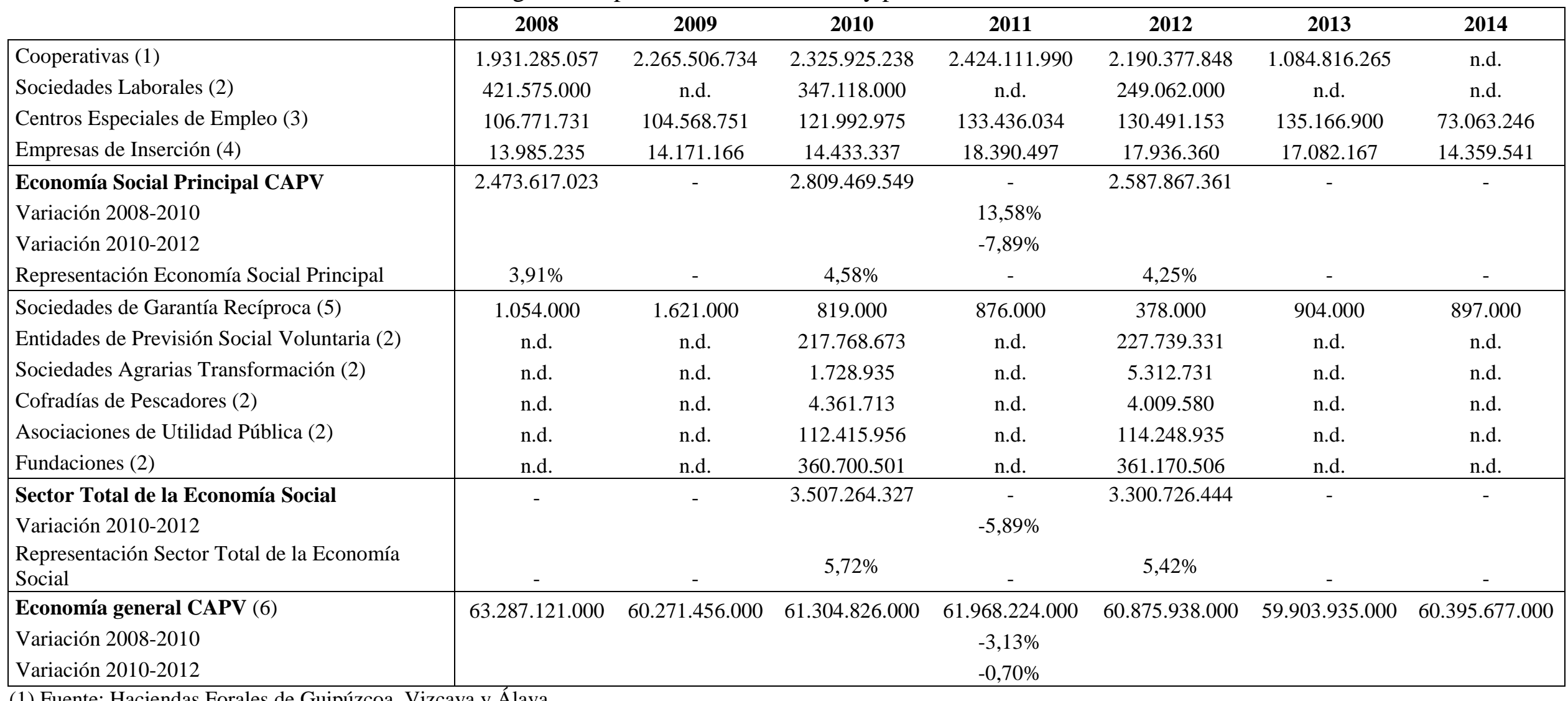

(1) Fuente: Haciendas Forales de Guipúzcoa, Vizcaya y Álava.

(2) Fuente: Dirección de Economía Social, Departamento de Empleo y Políticas Sociales, Gobierno Vasco (2008, 2010, 2012).

(3) Fuente: EHLABE - Asociación de Entidades de Trabajo Protegido del País Vasco.

(4) Fuente: GIZATEA- Asociación de Empresas de Inserción del País Vasco.

(5) Fuente: Oinarri, S.G.R.

(6) Economía General CAPV: Valor Añadido Bruto total generado en la Comunidad Autónoma del País Vasco. Fuente: EUSTAT-Instituto Vasco de Estadística. 
De esta forma, como se observa claramente en el gráfico 3, entre 2008 y 2010, la Economía Social Principal ha aumentado su capacidad de generación de VAB en un $13,58 \%$, mientras que en la economía general vasca ha caído un 3,13\%. Por el contrario, entre 2010 y 2012, la Economía Social Principal ha experimentado un descenso del $7,89 \%$ en el VAB generado y, en cambio, el VAB generado por la economía general vasca sólo ha descendido un $0,70 \%$.

Gráfico 3. Comparativa de las variaciones porcentuales en el empleo de la Economía Social Principal y de la Economía General de la CAPV (2008-2010 y 2010-2012).

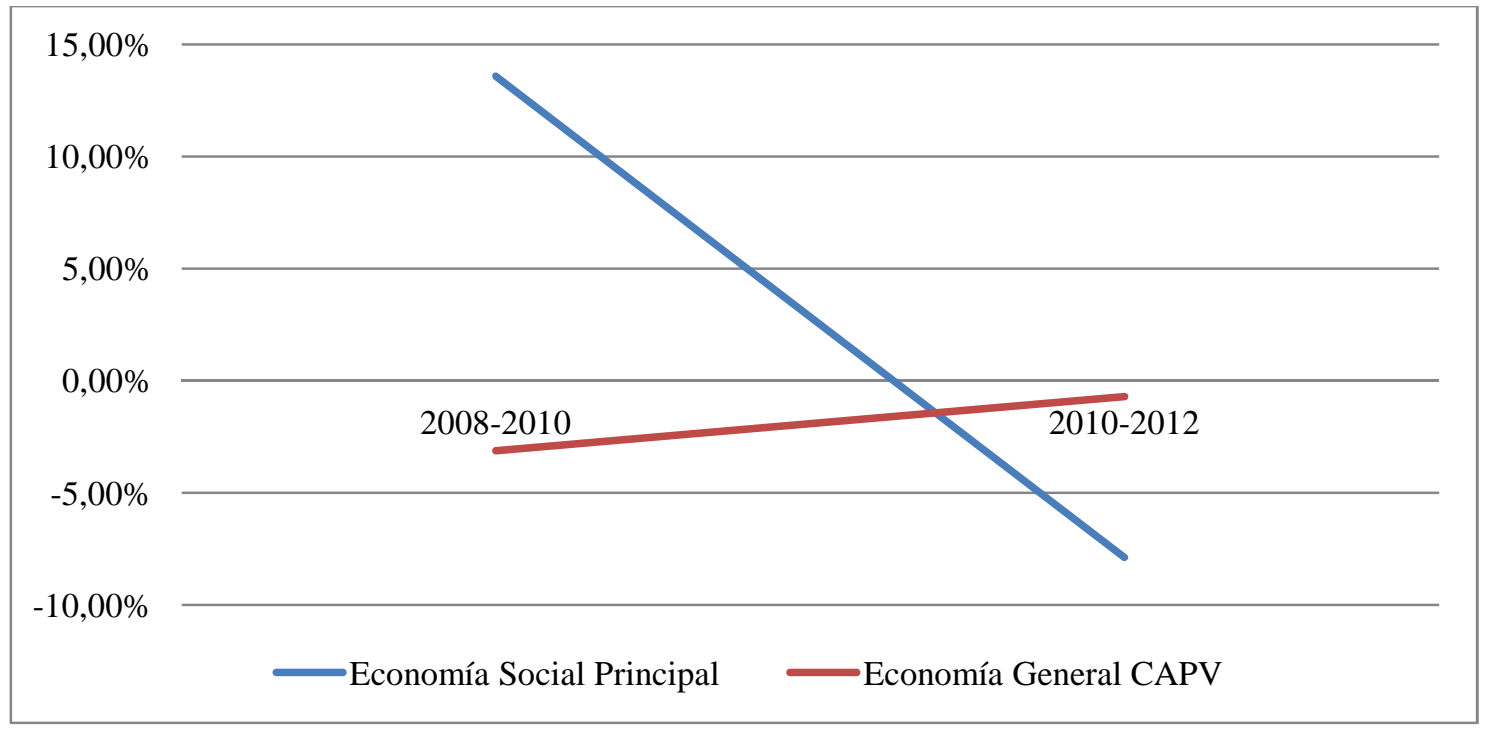

Fuente: elaboración propia

Por otra parte, atendiendo a la evolución del VAB generado por el Sector Total de la Economía Social, se observa que ha caído entre 2010 y 2012 un 5,89\%, dato mejor que el descenso producido entre dichos años por la Economía Social Principal (-7,89\%) pero que, sin embargo, es peor que el presentado por la economía general vasca en dicho periodo $(-0,70 \%)$. Esta evolución queda reflejada en el hecho de que el VAB generado por la economía social ha pasado de suponer el $5,72 \%$ del total del VAB generado en la CAPV en el año 2010, a representar el 5,42\% en 2012.

\subsection{Evolución de las políticas públicas en materia de Economía Social en el País Vasco durante la crisis económica}

Por otra parte, en el análisis de la respuesta de la economía social al escenario económico, resulta de interés analizar la evolución las políticas públicas en materia de Economía Social en el País Vasco durante la crisis económica, más aún teniendo en 
cuenta, como se ha señalado anteriormente, la creciente atención institucional recibida por el sector.

Existen diversas clasificaciones que difieren, no tanto en los contenidos, sino en la mera jerarquización (Bakaikoa y Morandeira, 2012: 237), diferenciando entre las políticas públicas sobre la economía social que inciden sobre la estructura del sector apoyando la creación y el desarrollo de las entidades, y las políticas de empleo que inciden directamente en el empleo de las entidades de economía social (Chaves, 2002, 2008). Atendiendo a estas clasificaciones, analizamos las acciones presupuestarias dentro de las medidas económicas de las políticas hard de oferta, es decir, los presupuestos dirigidos a las estructuras de las entidades de Economía Social.

La Administración Pública vasca cuenta con un programa específico para la Economía Social (Programa 3212 Economía Social) gestionado por la Dirección de Economía Social del Gobierno Vasco y que, en términos de subvenciones, se ha ido ajustando a lo largo de la crisis. En la siguiente tabla y gráfico, se recoge la evolución presupuestaria entre 2008 y 2014 de las ayudas gestionadas por las diferentes instancias: presupuesto general del Gobierno Vasco en su conjunto, del departamento al que pertenece la Dirección de Economía Social (es decir, del Departamento de Empleo y Políticas Sociales) y de la propia Dirección de Economía Social.

Como se puede observar, a pesar de que todas las instancias han sufrido descensos en los presupuestos, es la Dirección de Economía Social la que más ha acusado ese descenso. Por tanto, el fomento de la Economía Social no ha tenido el apoyo equivalente a la evolución de los recursos disponibles para todas las actividades a desarrollar por parte del Gobierno Vasco.

Tabla 4. Evolución de los presupuestos del Gobierno Vasco dedicados a la Economía Social para cada instancia (miles de euros). Periodo 2008-2014.

\begin{tabular}{|l|ccccccc|}
\cline { 2 - 8 } \multicolumn{1}{c|}{} & $\mathbf{2 0 0 8}$ & $\mathbf{2 0 0 9}$ & $\mathbf{2 0 1 0}$ & $\mathbf{2 0 1 1}$ & $\mathbf{2 0 1 2}$ & $\mathbf{2 0 1 3}$ & $\mathbf{2 0 1 4}$ \\
\hline $\begin{array}{l}\text { Dirección de } \\
\begin{array}{l}\text { Economía } \\
\text { Social }\end{array}\end{array}$ & $6.507,66$ & $6.712,03$ & $5.751,90$ & $5.492,07$ & $4.087,84$ & $3.576,00$ & $3.813,43$ \\
\hline $\begin{array}{l}\text { Departamento } \\
\text { de Empleo y } \\
\text { Pol. Sociales }\end{array}$ & 571.235 & 667.979 & 624.123 & 998.743 & 926.740 & 796.024 & 953.608 \\
\hline $\begin{array}{l}\text { Presupuestos } \\
\text { Generales }\end{array}$ & 9.939 .662 & 10.487 .458 & 10.315 .210 & 10.549 .489 & 10.449 .167 & 9.316 .657 & 10.215 .506 \\
\hline
\end{tabular}

Fuente: elaboración propia a partir de los Presupuestos Generales de la CAPV. 
Gráfico 4. Variación porcentual de los presupuestos del Gobierno Vasco dedicados a la Economía Social para cada instancia (2008-2014).

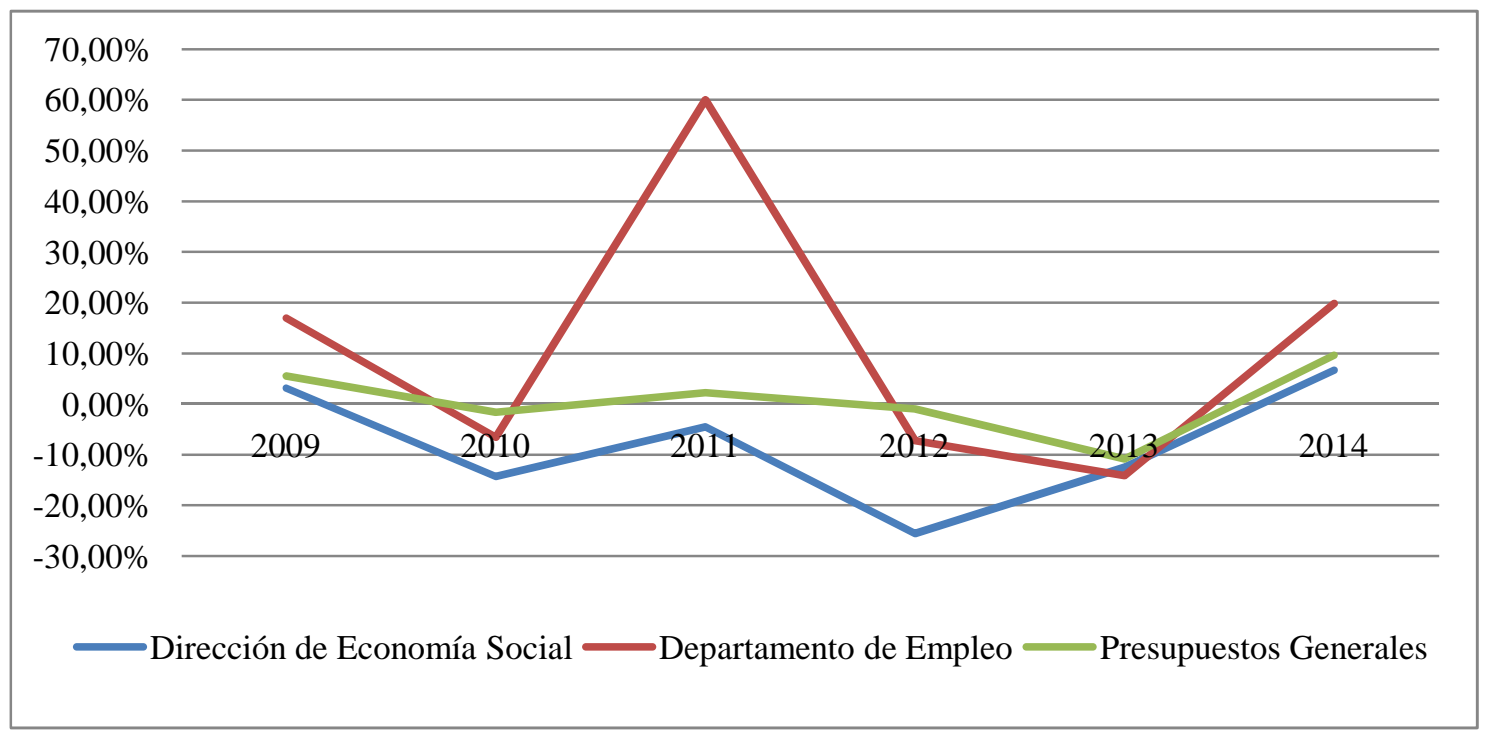

Fuente: elaboración propia.

\section{CONCLUSIONES}

La investigación aquí presentada sugiere que, en términos generales, la economía social ha respondido mejor ante la crisis económica en el País Vasco de lo que lo ha hecho la economía general. La Economía Social Principal, aun presentando ligeras variaciones negativas, ha sido capaz de mantener mejor el número de empresas y el empleo en todos los años, excepto 2009 y 2012. En cambio, la economía social no ha presentado una respuesta tan sólida a la crisis a nivel económico (medido a través del VAB generado). En ello puede haber influido, al menos parcialmente, el hecho de que la economía social antepone el empleo a los resultados económicos (OECD, 2013). En cualquier caso, los resultados obtenidos por la economía social durante la crisis se han alcanzado en un contexto en el que se han producido claros descensos en los presupuestos del Gobierno Vasco dedicados al sector. Ello pone en relieve la autosuficiencia de la economía social en su capacidad de respuesta a la crisis, desmitificando el hecho de que la economía social se sustente en una gran intervención de las instancias públicas (Savall, 2015).

Es significativo destacar que, tomando como referencia el Sector Total de la Economía Social, se observa una evolución entre 2010 y 2012 más positiva que la presentada por la Economía Social Principal. Es decir, cuando analizamos el conjunto 
de entidades que forman parte del sector, y no sólo algunas de ellas como cooperativas o sociedades laborales, el comportamiento de la economía social ante la crisis es mejor. Esto ratifica la importancia de contar con una conceptualización sólida e integradora de la economía social y de las entidades que la conforman, en tanto que fortalece, como aquí se demuestra, la respuesta de la economía social en su conjunto ante la crisis.

Las limitaciones de la investigación, derivadas de la falta de datos sobre todas las entidades de la economía social para el periodo completo 2008-2014, no han permitido realizar una valoración global más exacta de la evolución de la economía social durante la crisis. Ello pone de manifiesto las dificultades existentes para disponer de datos completos, homogéneos y continuados sobre la economía social vasca (Bretos, 2015), lo que lleva a diagnosticar un claro incumplimiento de la Ley 5/2011 de Economía Social (Pérez de Uralde, 2014) al menos en lo que se refiere a 'garantizar una información estadística de dichas entidades así como de sus organizaciones de representación, periódicamente actualizada', tal y como se expresa en su Disposición Adicional primera. Como señalan Chaves et al. (2013), disponer de estadísticas y herramientas para cuantificar la economía social es un aspecto fundamental para lograr la plena incorporación de esta realidad social al imaginario social del mundo político, académico y de la sociedad civil organizada.

En definitiva, los resultados de nuestra investigación han contribuido en esta dirección. Por primera vez en la literatura, se ha mostrado un análisis del conjunto del sector de la economía social, empleando datos inéditos obtenidos tanto de la Administración Pública como del sector privado que, si bien incompletos, son significativos y fiables. Mediante una estadística descriptiva básica, se han proporcionado resultados solventes que constatan que la economía social vasca ha sido capaz de resistir la crisis en mejores condiciones que la economía general. Sin duda, el haber podido disponer de datos completos y continuados sobre todas las entidades de la economía social hubiera enriquecido la investigación ya que, presumiblemente, se hubieran podido obtener unas conclusiones incluso más contundentes que las aquí presentadas acerca del comportamiento de la economía social ante la crisis. Parece por tanto necesario que la investigación futura continúe realizando esfuerzos en esta línea, proporcionando análisis sobre el conjunto de entidades que conforman el sector de la economía social, en aras de seguir fortaleciendo la visibilidad social del mismo y de 
dotarle de un mayor poder de negociación frente a las administraciones públicas, especialmente en contextos de crisis como el actual, en los que la economía social realiza una labor vital.

\section{BIBLIOGRAFÍA}

BAKAIKOA, B. y MORANDEIRA, J. (2012) El cooperativismo vasco y las políticas públicas. Ekonomiaz, no 79, pp. 235-263.

BARTLETT, W. y PRIDHAM, G.S. (1991) Cooperative Enterprises in Italy, Portugal and Spain: History, Development and Prospects. Journal of Interdisciplinary Economics, $\mathrm{n}^{\circ} 4$.

BAREA, J. y MONZÓN, J.L. (2008) Economía social e inserción laboral de las personas con discapacidad en el País Vasco. Bilbao: Fundación BBVA.

BEN-NER, A. (1988) Comparative Empirical Observations on Worker-owned and Capitalist Firms. International Journal of Industrial Organization, $\mathrm{n}^{\circ}$ 6, pp. 7-31.

BEN-NER, A., REN, T., y FLINT PAULSON, D. (2011) A sectoral comparison of wage levels and wage inequality in human services industries. Nonprofit and Voluntary Sector Quarterly, n 40, pp. 608-633.

BHOWMIK, S. y SARKER, K. (2002) Worker cooperatives as alternative productions systems. Work and Occupations, n 29 , pp. 460-482.

BRETOS, I. (2015) Medición y Visibilización de la Economía Social en el País Vasco: Dificultades, Aproximaciones y Propuestas desde el Observatorio Vasco de Economía Social. REVES-Revista Vasca de Economía Social, nº12 (próxima publicación).

BUENDIA, I. y GARCIA J.V. (2003): Cooperativismo y desarrollo local. Barcelona: Editorial Centro de Estudios Ramón Areces.

BURDÍN, G., y DEAN, A. (2009) New evidence on wages and employment in worker cooperatives compared with capitalist firms. Journal of Comparative Economics, $\mathrm{n}^{\circ}$ 37, pp. 517-533.

BIRCHALL, J. (2010) People-centred businesses: Co-operatives, mutuals and the idea of membership. Basingstoke: Palgrave Macmillan.

BIRCHALL, J. (2013) The potential of co-operatives during the current recession: theorizing comparative advantage. Journal of Entrepreneurial and Organizational Diversity, ${ }^{\circ}$ 2, vol. 1, pp. 1-22. 
BIRCHALL, J. y KETILSON, L. (2009) Resilience of the Cooperative Business Model in Times of Crisis. Geneva: International Labor Organization.

CALDERÓN, B. y CALDERÓN, M.J. (2012) La calidad del empleo de las entidades de la economía social en periodos de crisis. Ekonomiaz, Revista Vasca de Economía, $\mathrm{n}^{\circ}$ 79, pp. 31-57.

CARINI, C. y CARPITA, M. (2014) The impact of the economic crisis on Italian cooperatives in the industrial sector. Journal of Co-operative Organization and Management, $\mathrm{n}^{\circ}$ 2, pp. 14-23.

CASTRO, R. SANTERO, R. MARTÍNEZ, M.I. y GUILLÓ, N. (2013) Impacto socioeconómico de las empresas de economía social en España. Una valoración cuantitativa de sus efectos sobre la cohesión social. CIRIEC-España, Revista de Economía Pública, Social y Cooperativa, n ${ }^{\circ}$ 79, pp. 35-58.

CHAVES, R. (2002) Politiques Publiques et Economie Sociale en Europe: le cas de l'Espagne. Annals of Public and Cooperative Economics, $\mathrm{n}^{\circ}$ 73, pp. 453-480.

CHAVES, R. (2008) Public Policies and Social Economy in Spain and Europe. CIRIEC-España, Revista de Economía Pública, Social y Cooperativa, $\mathrm{n}^{\circ}$ 62, pp. 3560.

CHAVES, R. y MONZON, J.L. (2008) La Economía Social en la Unión Europea. Bruselas: Comité Económico y Social Europeo.

CHAVES, R. y MONZON, J.L. (2012) Beyond the crisis: the social economy, prop of a new model of sustainable economic development. Service Business, nº 6, pp. 5-26.

CHAVES, R., MONZÓN, J.L., PÉREZ DE URALDE, J.M. y RADRIGÁN, M. (2013) La Economía Social en Clave Internacional. Cuantificación, Reconocimiento Institucional y Visibilidad Social en Europa, Iberoamérica y Norte de África, REVESCO. Revista de Estudios Cooperativos, $\mathrm{n}^{\circ}$ 112, pp. 122-150.

COMISIÓN EUROPEA (2013) EaSi, Nuevo Programa Marco de la UE para el Empleo y la Política Social. Luxemburgo.

CONSEJO EUROPEO (2015) La promoción de la economía social como motor clave del desarrollo económico y social en Europa (15071/15), N. ${ }^{\circ}$ doc. prec.: 13766/15 SOC 643 EMPL 423, Bruselas.

CONTE, M. y JONES, D. (1991) On the entry of employee-owned firms: Theory and evidence from US manufacturing Industries, 1870-1960. Working paper No. 91/5, Hamilton College, Clinton, NY. 
CO-OPERATIVES UK (2014) The UK Co-operative Economy 2014: Untold Resilience. Manchester : Co-operatives UK.

DÍAZ M. y MARCUELLO, C. (2010) Impacto económico de las cooperativas. La generación de empleo en las sociedades cooperativas y su relación con el PIB. CIRIEC-España, Revista de Economía Pública, Social y Cooperativa, nº 67, pp. 2344.

EL PAÍS (2015) Cinco entidades de la economía social vasca promueven empleo de calidad. Bilbao: El País. Consultado el 12/05/2015. Disponible en:

www.ccaa.elpais.com/ccaa/2015/03/25/paisvasco/1427303844_953878.html

ERDAL, D. (2011) Beyond the corporation: Humanity working. London: The Bodley Head.

ERDAL, D. (2012) Employee ownership and health. Presentación en el congreso “Cooperatives and Public Health" (8 mayo de 2012). Edinburgh: Scottish Enterprise. ESTRIN, S. (1985) The Role of Producer Co-operatives in Employment Creation. Economic Analysis and Workers' Management, n 19, pp. 345-384.

ETXEZARRETA, E., y MORANDEIRA, J. (2012) Consideraciones conceptuales sobre la Economía Social a la luz de la Ley 5/2011. REVES-Revista Vasca de Economía Social, $\mathrm{n}^{\circ} 8$, pp. 7-36.

FAKHFAKH, F., PÉROTIN, V. y GAGO, M. (2012) Productivity, capital and labor in labor-managed and conventional firms. Industrial and Labor Relations Review, $\mathrm{n}^{\circ}$ 65 , pp. 847-879.

GOBIERNO VASCO (2008) Estadística de la Economía Social 2008. Departamento de Empleo y Políticas Sociales, Gobierno Vasco, Vitoria-Gasteiz.

GOBIERNO VASCO (2010) Estadística de la Economía Social 2010. Departamento de Empleo y Políticas Sociales, Gobierno Vasco, Vitoria-Gasteiz.

GOBIERNO VASCO (2012) Estadística de la Economía Social 2012. Departamento de Empleo y Políticas Sociales, Gobierno Vasco, Vitoria-Gasteiz.

GRÁVALOS, M.A. y POMARES, I. (2001) Cooperativas, Desempleo y Efecto Refugio. REVESCO. Revista de Estudios Cooperativos, nº 74, pp. 69-84.

MARCUELLO, C. (2008) Empresas de inserción: puentes de inclusión en el mercado laboral. Valencia: CIRIEC-España. 
MELIAN, A. y CAMPOS, V. (2010) Emprendedurismo y economía social como mecanismos de inserción socio laboral en tiempos de crisis. REVESCO. Revista de Estudios Cooperativos, $\mathrm{n}^{\circ} 100$, pp. 43-67.

MONZÓN, J.L. (2010a) Las grandes cifras de la Economía Social en España. Ámbito, entidades y cifras clave. Año 2008. Valencia: CIRIEC-España.

MONZÓN, J.L. (2010b) La Economía Social en España en el año 2008. Ámbito, magnitudes, actividades y tendencias, Valencia: CIRIEC-España.

MONZÓN, J.L. (2010c) Entidades no lucrativas y el Tercer Sector de Acción Social. En MONZÓN, J.L.: La Economía Social en España en el año 2008. Ámbito, magnitudes, actividades y tendencias. Valencia: CIRIEC, pp. 556-563.

MONZÓN, J.L. (2013) Empresas Sociales y Economía Social: perímetro y propuestas metodológicas para la medición de su impacto socioeconómico en la U.E. Revista de Economía Mundial, nº 35, pp. 151-164.

MONZON, J.L. y CHAVES, R. (2012) La Economía Social en la Unión Europea. Bruselas: Comité Económico y Social Europeo.

OECD (2013) Job Creation through the Social Economy and Social Entrepreneurship. Paris: OECD-Organization for Economic Co-operation and Development.

PARLAMENTO EUROPEO (2013) La contribución de las cooperativas a la salida de la crisis (2012/232/INI). Bruselas.

PARLAMENTO EUROPEO (2015) Emprendimiento Social e Innovación Social en la lucha contra el desempleo (2014/2236/INI). Bruselas.

PÉREZ DE MENDIGUREN, J.C., ETXEZARRETA, E. y GURIDI, L. (2009) Economía Social, Empresa Social y Economía Solidaria: diferentes conceptos para un mismo debate. Papeles de Economía Solidaria, ${ }^{\circ} 1$, REAS-Euskadi, Bilbao.

PÉREZ DE URALDE, J.M. (2014) La Inaplicación de la Ley de Economía Social en un Contexto de Crisis Económica. Algunas Interpretaciones y Propuestas, CIRIECEspaña, Revista de Economía Pública, Social y Cooperativa, nº 81, pp. 33-59.

PEROTIN, V. (2006) Entry, exit and the business cycle: Are cooperatives different? Journal of Comparative Economics, $\mathrm{n}^{\circ} 34, \mathrm{pp} .295-316$.

PEROTIN, V. (2013) Worker cooperatives: Good, sustainable jobs in the community. Journal of Entrepreneurial and Organizational Diversity, $\mathrm{n}^{\circ} 2$, pp. 34-47.

ROELANTS, B., HYUNGSIK, E. y TERRASY, E. (2014) Cooperatives and Employment: A Global Report. Bruselas: CICOPA. 
ROMÁN, C. (2014) Las Cooperativas Españolas y los Ciclos Económicos: un análisis Comparado. CIRIEC-España, Revista de Economía Pública, Social y Cooperativa, $\mathrm{n}^{\circ} 80$, pp. 77-109.

RONDINELLI, M. y SARATEANU, A. (2012) Riding the Economic Downturn: Cooperatives in Europe. En Dallago, B. and Guglielmetti, C. (2012) The Consequences of the International Crisis for European SMEs: Vulnerability and Resilience, New York: Routledge.

SALA, M., FARRÉ, M. y TORRES, T. (2014) Un análisis del comportamiento cíclico de las cooperativas y sociedades laborales españolas y de su relación con la actividad económica. REVESCO. Revista de Estudios Cooperativos, n 115 , pp. 729.

SAVALL, T. (2015) La Economía Social y las políticas públicas. Análisis del cambio político, elementos condicionantes y resultados. Tesis Doctoral, Universidad de Valencia.

SMITH, S. y ROTHBAUM, J. (2013) Cooperatives in a Global Economy: Key Economic Issues, Recent Trends, and Potential for Development. Policy Paper No. 68, Institute for the Study of Labor (IZA), Bonn, Alemania.

SPEAR, R. (1996) Cooperativas y Empleo en el Reino Unido. CIRIEC-España, Revista de Economía Pública, Social y Cooperativa, nº 15, pp. 25-31.

STIGLITZ, J. (2009) Moving beyond market fundamentalism to a more balanced economy. Annals of Public and Cooperative Economics, $\mathrm{n}^{\circ}$ 80, pp. 345-360.

THOMAS, A. (2004) The rise of social cooperatives in Italy. Voluntas, 15, 243-264.

VIETA M. (2013) The emergence of the "empresas recuperadas por sus trabajadores": A political economic and sociological appraisal of two decades of self-management in Argentina. Euricse Working Paper n. 55-13, Trento, Italia.

WESTERDAHL, S. y WESTLUND, H. (1998) Social Economy and New Jobs: a Summary of Twenty Case Studies on European Regions. Annals of Public and Cooperative Economics, $\mathrm{n}^{\circ}$ 69, pp. 193-218.

WILLIAMSON, T., IMBROSCIO, D. y ALPEROVITZ, G. (2003) Making a place for a community: local democracy in a global era. New York: Routledge.

ZEVI, A., ZANOTTI, A., SOUlAGE, F. y ZELAIA, A. (2011) Beyond the Crisis: Cooperatives, Work, Finance. Bruselas: CECOP. 